\title{
The preclinical pharmacological study on HX0969W, a novel water-soluble pro-drug of propofol, in rats
}

\author{
YuJun Zhang Equal first author, 1, 2 , YingYing Jiang Equal first author, 1 , HaiYan Wang ${ }^{3}$, Bin Wang ${ }^{4}$, Jun Yang ${ }^{1,2}$, Yi Kang ${ }^{1,2}$, Jun \\ Chen $^{5}$, Jin Liu ${ }^{1,2}$, Wen-sheng Zhang ${ }^{\text {Corresp. 1,2 }}$ \\ ${ }^{1}$ Department of Anesthesiology, Laboratory of Anesthesia and Critical Care Medicine, Translational Neuroscience Centre, West China Hospital, Sichuan \\ University, Chengdu, China \\ 2 \\ 2 National-Local Joint Engineering Research Center of Translational Medicine of Anesthesiology, West China Hospital, Sichuan University, Chengdu, China \\ 3 Department of Anaesthesiology, Yuebei People's Hospital, Guangdong University, Shaoguan, China \\ 4 Department of Anesthesiology, Guizhou Provincial People's Hospital, Guiyang, China \\ 5 Laboratory Department Guizhou Provincial Corps Hospital of PAPF, Guiyang, China \\ Corresponding Author: Wen-sheng Zhang \\ Email address: zhang_ws@scu.edu.cn
}

Background: Propofol is the most widely used intravenous sedative-hypnotic anesthetic in clinical practice. However, many serious side effects have been related to its lipid emulsion formulation. The prodrug design approach was used to develop the water-soluble propofol, which could effectively resolve the limitations associated with the lipid emulsion formulation. Thus, the new water-soluble pro-drug of propofol, HX0969W, was designed and synthesized. The objective of this study was to conduct preclinical pharmacological studies on this novel water-soluble pro-drug of propofol.

Methods: The assessment of the loss of the righting reflex (LoRR) was used for the pharmacodynamic study, and liquid chromatography-tandem mass spectrometry and high-performance liquid chromatography- fluorescence were used for the pharmacokinetic study.

Results: The potency of HX0969W (ED ${ }_{50}[95 \% \mathrm{Cl}], 46.49$ [43.89 - 49.29] mg/kg) was similar to that of fospropofol disodium (43.66 [43.57 - 43.75] mg/kg), but was lower than that of propofol (4.82 [4.81 $4.82 \mathrm{mg} / \mathrm{kg}$ ). Administered with a dose of 2 -fold $\mathrm{ED}_{50}$, propofol required a shorter time to cause LoRR than that of HX0969W and fospropofol. However, the LoRR duration was significantly longer in response to the administration of HX0969W and fospropofol disodium than that caused by propofol. In the pharmacokinetic study, the $\mathrm{C}_{\max }$ of fospropofol was higher than that of HX0969W. HX0969W had a shorter mean residual time and a rapid clearance rate than that of fospropofol disodium. There was no significant difference between the $T_{\max }$ of the propofol whether it was released by HX0969W or fospropofol disodium; the $\mathrm{C}_{\max }$ of propofol released by HX0969W was similar to that of propofol, which was higher than the propofol released by fospropofol disodium. 
1 Manuscript Title: The preclinical pharmacological study on HX0969W, a novel water-soluble

2 pro-drug of propofol, in rats

3 YuJun Zhang, 1, 2, \#, YingYing Jiang, 1, \#, HaiYan Wang, ${ }^{3}$, Bin Wang, ${ }^{4}$, Jun Yang, ${ }^{\text {, Yi Kang, }}{ }^{1}$,

4 Jun Chen, ${ }^{5}$, Jin Liu, ${ }^{1,2}$, WenSheng Zhang, 1, 2, *

5 1: Department of Anesthesiology, Laboratory of Anesthesia and Critical Care Medicine,

6 Translational Neuroscience Center, West China Hospital, Sichuan University, Chengdu 610041,

7 China.

8 2: National-Local Joint Engineering Research Center of Translational Medicine of

9 Anesthesiology, West China Hospital, Sichuan University, Chengdu 610041, China.

10 3: Department of Anaesthesiology, Yuebei People's Hospital, Guangdong University, Shaoguan,

11 Guangdong 512026, China.

12 4: Department of Anesthesiology, Guizhou Provincial People's Hospital, Guiyang 550002, China.

13 5: Laboratory Department Guizhou Provincial Corps Hospital of PAPF, Guiyang, Guizhou

14550000 , China.

$15{ }^{\#}$ These authors contributed equally to this work.

$16 *$ Correspondence author:

17 Wensheng Zhang

18 Laboratory of Anesthesia and Critical Care Medicine, Department of Anesthesiology,

19 Translational Neuroscience Center, West China Hospital, Sichuan University, Chengdu 610041,

20 China.

21 E-mail: zhang_ws@scu.edu.cn 


\section{Abstract}

Background: Propofol is the most widely used intravenous sedative-hypnotic anesthetic in clinical practice. However, many serious side effects have been related to its lipid emulsion formulation. The pro-drug design approach was used to develop the water-soluble propofol, which could effectively resolve the limitations associated with the lipid emulsion formulation. Thus, the new water-soluble pro-drug of propofol, HX0969W, was designed and synthesized. The objective of this study was to conduct preclinical pharmacological studies on this novel water-soluble pro-drug of propofol.

Methods: The assessment of the loss of the righting reflex (LoRR) was used for the pharmacodynamic study, and liquid chromatography-tandem mass spectrometry and highperformance liquid chromatography- fluorescence were used for the pharmacokinetic study.

Results: The potency of HX0969W (ED ${ }_{50}[95 \% \mathrm{CI}], 46.49$ [43.89 - 49.29] mg/kg) was similar to that of fospropofol disodium $(43.66[43.57-43.75] \mathrm{mg} / \mathrm{kg})$, but was lower than that of propofol $(4.82[4.81-4.82] \mathrm{mg} / \mathrm{kg})$. Administered with a dose of 2-fold $\mathrm{ED}_{50}$, propofol required a shorter time to cause LoRR than that of HX0969W and fospropofol. However, the LoRR duration was significantly longer in response to the administration of HX0969W and fospropofol disodium than that caused by propofol. In the pharmacokinetic study, the $\mathrm{C}_{\max }$ of fospropofol was higher than that of HX0969W. HX0969W had a shorter mean residual time and a rapid clearance rate than that of fospropofol disodium. There was no significant difference between the $T_{\max }$ of the propofol whether it was released by HX0969W or fospropofol disodium; the $\mathrm{C}_{\max }$ of propofol released by HX0969W was similar to that of propofol, which was higher than the propofol 
43 released by fospropofol disodium. 
44

45

46

47

\section{Introduction}

General anesthesia is a combination of drugs, including sedative-hypnotic agents, analgesics, or muscle relaxants, which put the patients in a sleep-like state before surgery or other medical examinations. Propofol is the most widely used intravenous sedative-hypnotic anesthetic in clinical practice (Hemphill et al. 2019). However, many serious side effects have been related to its lipid emulsion formations, such as emulsion instability, injection pain, hyperlipidemia, infection, fat metabolism disorder, and propofol-related infusion syndrome (Diaz et al. 2014; Lee et al. 2014; Mirrakhimov et al. 2015; Pestana et al. 1996; Prankerd \& Stella 1990; Singh et al. 2011; Wachowski et al. 1999; Wolf et al. 2001; Zhou et al. 2015). The pro-drug design approach was widely used to develop the novel water-soluble propofol, which could effectively avoid the limitations associated with its lipid emulsion formulation (Feng et al. 2017).

Fospropofol disodium is the first water-soluble pro-drug of propofol that has been approved by the U.S. Food and Drug Administration to be administered as a bolus injection for adult patients undergoing diagnostic or therapeutic procedures (Moore et al. 2009; Telletxea et al. 2012). Although it is confirmed that formaldehyde, which is one of the metabolites of

fospropofol disodium, does not accumulate after a single administration (Garnock-Jones \& Scott 2010; Kumpulainen et al. 2008), it is still considered a potentially risk for systemic toxicity and has limited the clinical indication of fospropofol disodium for continuous infusion. A new watersoluble pro-drug of propofol was designed and synthesized in our laboratory (patent number, WO2011160268; denoted as HX0969W), which was metabolized to propofol, $\gamma$-hydroxybutyrate (GHB), and phosphate in vivo. GHB was found to be further converted into carbon dioxide and 
65

66

67

68

69

water (Lang et al. 2014; Rousseau et al. 2012). In contrast to fospropofol disodium, HX0969W cloud produce an effective sedative-hypnotic effect with lesser potential risks of systemic toxicity.

In the present study, we evaluated the median effective dose $\left(\mathrm{ED}_{50}\right)$ of $\mathrm{HX} 0969 \mathrm{~W}$, fospropofol disodium, and propofol for the loss of righting reflex (LoRR) in rats using the up and down method. Then, we measured the onset time and duration for HX0969W, fospropofol disodium and propofol after single intravenous injections. Furthermore, the pharmacokinetic parameters of the drugs were assessed in rats.

\section{Material and Methods}

\subsection{Materials}

HX0969W and fospropofol disodium were synthesized at Yichang Humanwell Pharmaceutical Co., Ltd. (Yichang, China). Propofol was purchased from AstraZeneca (Shanghai, China). 7Hydroxycoumarin, thymol, and ammonium acetate were purchased from Sigma-Aldrich Co., Ltd. (MO, USA). Acetonitrile and methanol were obtained from ROE Scientific Inc. (DE, USA). Ultrapure water was produced using Milli- ${ }^{\circledR}{ }^{\circledR}$ integral water purification system (Merck Millipore, Germany).

\subsection{Experimental animals}

Adult Sprague-Dawley rats (age: 8 - 10 weeks, body weight: 220 - 350g) were purchased from

Dossy Biological Technology Co., Ltd. (Chengdu, China) and housed in polypropylene cages (less than 5 animals per cage) at the Experimental Animal Center of Sichuan University (Chengdu, China) at an ambient temperature of $25 \pm 1{ }^{\circ} \mathrm{C}$, a controlled humidity of $50 \%-70 \%$, 
and a 12-h light-dark cycle (7 a.m. to 7 p.m.). The rats were provided with a radiation-sterilized commercial diet and filtered water ad libitum. The rats were subjected to fasting for $12 \mathrm{~h}$ with uncontrolled water supply prior to initiation of dosing; food was supplied immediately after dosing. The experimental interventions and sample collections in this study were accomplished through the tail vein after venipuncture (Terumo ${ }^{\circledR}$ intravenous catheter; 24G, Tokyo, Japan). Thus, no anesthetic procedure was used in this study. At the end of experiments, all rats were euthanized via overdose of pentobarbital sodium. All experiments were performed with the approval of the Committee of Scientific Research and the Institutional Animal Experimental Ethics Committee of West China Hospital, Sichuan University, Chengdu, China (2015015A).

\subsection{Measurement of the hypnotic median effective dose}

The $\mathrm{ED}_{50}$ values of HX0969W, fospropofol disodium, and propofol were determined by the up and down method (Dixon 1991; Glen \& Hunter 1984; Kilpatrick et al. 2007). The tail vein was cannulated with a Terumo ${ }^{\circledR}$ intravenous catheter (24G; Tokyo, Japan) for drug administration. After administered the drug, the rats were assessed one by one in separate box for the LoRR effect (once the rat lay prone and no reaction to mild stimuli, the rat was gently changed to the supine position recognized as LoRR), and were provided with warm blanket and oxygen inhalation to reduce external stimulation. If the duration of LoRR after drug administration was more than $30 \mathrm{~s}$, the next rat was administered with a lower dose. On the contrary, if the duration of LoRR was less than $30 \mathrm{~s}$ (none-LoRR), a higher dose was injected in the next rat. Recording the LoRR to none-LoRR as a cross, the measurement of the $\mathrm{ED}_{50}$ value was terminated once more than five crosses occurred. An accurate $\mathrm{ED}_{50}$ value with $95 \%$ confidence intervals $(\mathrm{CI})$ was 
107

108

109

110

111

112

113

114

115

116

117

118

119

120

121

122

123

124

125

126

127

calculated using standard computing equations (Dixon 1991; Glen \& Hunter 1984; Kilpatrick et al. 2007).

\subsection{Pharmacodynamic study in rats}

Thirty adult Sprague-Dawley rats were randomly divided into three groups to evaluate the efficacy of HX0969W, fospropofol disodium, and propofol $(n=10$ in each group, 5 males and 5 females). The drugs were administered via tail vein through the Terumo ${ }^{\circledR}$ intravenous catheter (24G; Tokyo, Japan) at equivalent doses (2-fold dose of $\mathrm{ED}_{50}$ for LoRR in rats) at an injection speed of $0.25 \mathrm{~mL} / \mathrm{s}$. Then, each rat was placed in a separate box with a warm blanket and oxygen inhalation. For the pharmacodynamic study, the time to LoRR (onset time) and time to recovery from LoRR (duration) were recorded. All rats were observed closely for any mortality, behavioural changes, and clinical symptoms of toxicity.

\subsection{Pharmacokinetic study in rats}

Before the experiment, the rats were subjected to fasting for $12 \mathrm{~h}$ with uncontrolled water supply; food was supplied immediately after drug administration. The tail vein was cannulated with a Terumo ${ }^{\circledR}$ intravenous catheter (24G; Tokyo, Japan) for drug administration and sample collection. HX0969W, fospropofol disodium, and propofol were injected at their equivalent doses in the rats through their tail veins ( $\mathrm{n}=10$ in each group, 5 males and 5 females). The blood samples from the rats were collected at different times, which were determined based on the results of the preliminary experiments. For HX0969W and fospropofol disodium, $50 \mu \mathrm{L}$ blood samples were collected at $1,2,3,4,5,7,10,15,20,30,45,60,90,120,180$, and 240 min after drug administration. For propofol, $50 \mu \mathrm{L}$ blood samples were collected at $0.5,1,2,3,5,7,10,15$, 
128

129

130

131

132

133

134

135

136

137

138

139

140

141

142

143

144

145

146

147

148

$20,30,45,60,90,120$, and 180 min after drug administration.

\subsubsection{Sample preparation and quantitative method}

Blood samples from the HX0969W and fospropofol disodium groups were deproteinized by

methanol solution with 7-Hydroxycoumarin (internal standard, IS). The mixture was centrifuged

for $10 \mathrm{~min}$ at $25,000 \times \mathrm{g}$ at $4{ }^{\circ} \mathrm{C}$ after vortexing. The supernatant was then subjected to

determination and quantification by liquid chromatography-tandem mass spectrometry (LC-

MS/MS). The LC-MS/MS analysis system consisted of an Agilent 6460 triple quadrupole mass spectrometer (Agilent Technologies, CA, USA) with an electrospray ionization source.

Chromatographic separation was performed using a Zorbax eclipse plus C8 column $(100 \mathrm{~mm} \times$

$2.1 \mathrm{~mm}, 3 \mu \mathrm{m}$ ) at $12{ }^{\circ} \mathrm{C}$ with $5 \mathrm{mM}$ ammonium acetate in deionized water and acetonitrile at a volume ratio of $65: 35$ for $\mathrm{HX} 0969 \mathrm{~W}$ and $70: 30$ for fospropofol disodium, at a flow rate of 0.3

$\mathrm{mL} / \mathrm{min}$. The mass spectrometry conditions were as follows: negative ionization mode; the

sheath gas flow rate, $5.0 \mathrm{l} / \mathrm{min}$; sheath gas heater temperature, $350{ }^{\circ} \mathrm{C}$; nebulizer pressure, $30 \mathrm{psi}$;

capillary voltage, $4,500 \mathrm{~V} ; m / z 343.0 \rightarrow 177.0$ for $\mathrm{HX} 0969 \mathrm{~W}, m / z 287.00 \rightarrow 177.00$ for

fospropofol disodium, and $m / z \quad 160.7 \rightarrow 89.0$ for IS.

For propofol, the samples were deproteinized with a methanol solution with thymol (IS).

4 The mixture was centrifuged for $10 \mathrm{~min}$ at $25,000 \times \mathrm{g}$ at $4{ }^{\circ} \mathrm{C}$ after vortexing. The supernatant was then subjected to determination and quantification by high-performance liquid chromatography- fluorescence. The analysis system consisted of an Agilent Zorbax XDB (Agilent Technologies, CA, USA) equipped with a C18 column $(150 \times 4.6 \mathrm{~mm}, 5 \mu \mathrm{m})$ and a fluorescence detector. The liquid chromatography conditions were as follows: mobile phase 
solvent, acetonitrile and water at the volume ratio of $40: 60$ at a flow rate of $1.2 \mathrm{~mL} / \mathrm{min}$;

fluorescence detector, the wavelength of excitation and emission at 276 and $310 \mathrm{~nm}$, respectively.

The method validation results were detailed in the supplementary material includes the following parameters: specificity, linearity, lower limit of quantitation (LLOQ), precision, accuracy, matrix effect, extraction recovery, stability and dilution integrity.

\subsubsection{Pharmacokinetic analysis}

Noncompartmental pharmacokinetic methods were used for pharmacokinetic analysis of HX0969W, fospropofol disodium, and propofol using the Phoenix Winnonlin ${ }^{\circledR}$ software (version 6.3, NJ, USA). The pharmacokinetic parameters used in this study were as follows: maximum concentration $\left(\mathrm{C}_{\max }\right)$ and the time to acquire $\left(\mathrm{T}_{\max }\right)$, area under curve (AUC) from zero to the last time point $\left(\mathrm{AUC}_{0-\mathrm{t}}\right)$, mean residual time (MRT), half-time $\left(\mathrm{t}_{1 / 2}\right)$, and clearance $(\mathrm{CL})$.

\subsection{Data Analysis}

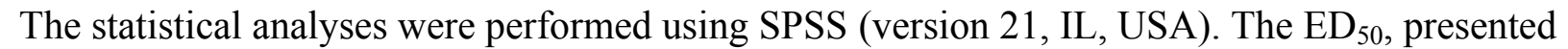
as mean and 95\% CI, was calculated using the up and down method. The Kruskal- Wallis test followed by the Mann-Whitney U test was used for the data that did not fit normality, and the one-way $A N O V A$ followed by Tukey's test was used for the data that did not fit normality and equality of variance. The level of statistical significance was set at $\mathrm{p}<0.05$.

\section{Results}

\subsection{Measurement of the median effective dose for the sedative-hypnotic effect}

Following a single intravenous administration of the drug, each rat was placed in a separate box to record the time to LoRR and time to recovery from LoRR. Then, the median effective dose to 
170

171 (Dixon 1991; Glen \& Hunter 1984; Kilpatrick et al. 2007). The potency of HX0969W in rats

$172\left(\mathrm{ED}_{50}[95 \% \mathrm{CI}], 46.49\right.$ [43.89 - 49.29] $\left.\mathrm{mg} / \mathrm{kg}\right)$ was found to be similar to that of fospropofol

173

174

175

176

177

178

179

180

181

182

183

achieve the sedative-hypnotic effect (Table 1) was calculated using the up and down method disodium $\left(\mathrm{ED}_{50}[95 \% \mathrm{CI}], 43.66[43.57\right.$ - 43.75$\left.] \mathrm{mg} / \mathrm{kg}\right)$, but lower than that of propofol $\left(\mathrm{ED}_{50}\right.$ [95\% CI], $4.82[4.81-4.82] \mathrm{mg} / \mathrm{kg}$ ). Due to the pro-drug design, HX0969W and fospropofol disodium are metabolized to propofol, which produces the sedative-hypnotic effect. Thus, the dosages of HX0969W and fospropofol used for general anesthesia were higher than those of propofol.

\subsection{Pharmacodynamic study in rats}

After administering a 2-fold $\mathrm{ED}_{50}$ dose for LoRR, the time to LoRR (onset time) and time to recovery from LoRR (duration) were measured in the rats. No mortality or significant clinical symptoms of toxicity were observed in these rats. As for the onset time (Fig. 1A), propofol (0.4 $\pm 0.1 \mathrm{~min})$ required a shorter time to cause LoRR than that required for HX0969W $(1.8 \pm 0.4$ $\min , p=0.017)$ and fospropofol disodium $(2.1 \pm 0.7 \mathrm{~min}, \mathrm{p}=0.006)$. However, the duration of LoRR for HX0969W $(75.8 \pm 9.6 \mathrm{~min}, \mathrm{p}=0.036)$ and fospropofol disodium $(68.5 \pm 18.4 \mathrm{~min}, \mathrm{p}=$ $0.041)$ were significantly longer than that of propofol (27.1 $\pm 6.0 \mathrm{~min}$, Fig. 1B).

\subsection{Pharmacokinetic study of HX0969W and fospropofol disodium in rats}

After a single intravenous injection, there was no significant difference in the action onset time and duration between HX0969W and fospropofol disodium. The efficacy of a drug is closely related to its pharmacokinetic features. Therefore, we measured the concentration-time profiles of HX0969W and fospropofol disodium in rats. The concentration-time curves are presented in 
191 Fig. 2, and the calculated pharmacokinetic parameters are shown in Table 2. Following the 192 administration of equivalent doses $(92.98 \mathrm{mg} / \mathrm{kg}$ for $\mathrm{HX} 0969 \mathrm{~W}, 87.32 \mathrm{mg} / \mathrm{kg}$ for fospropofol 193 disodium), the maximal concentration of fospropofol disodium $(603.49 \pm 411.29 \mu \mathrm{g} / \mathrm{mL})$ was 194 found to be higher than that of HX0969W $(321.30 \pm 67.22 \mu \mathrm{g} / \mathrm{mL})$. In rat plasma, HX0969W

had a shorter mean residual time $(3.67 \pm 1.71 \mathrm{~min})$ and a rapid clearance rate $(89.97 \pm 15.94$ $\mathrm{ml} / \mathrm{min} / \mathrm{kg}$ ) than those of fospropofol disodium $(13.15 \pm 5.45 \mathrm{~min}$ and $31.12 \pm 19.09 \mathrm{ml} / \mathrm{min} / \mathrm{kg}$, respectively). The computed AUC value of $\mathrm{HX} 0969 \mathrm{~W}$ from zero to the last time point was $1053.78 \pm 214.37 \mathrm{~min}^{*} \mu \mathrm{g} / \mathrm{ml}$, which was less than that of fospropofol disodium $(3804.92 \pm$ $\left.2091.75 \mathrm{~min}^{*} \mu \mathrm{g} / \mathrm{ml}\right)$. Thus, we considered that HX0969W, after a bolus injection in rats, had a faster metabolism in vivo as compared to fospropofol disodium. The pharmacokinetic parameters of HX0969W and fospropofol disodium could not explain the lack of significant differences in the onset time and duration. Therefore, we further evaluated the concentration of propofol released by HX0969W and fospropofol disodium in rats.

\subsection{Pharmacokinetic study of propofol in rats}

Using an equivalent dose of HX0969W, fospropofol disodium and the parent drug propofol, the concentration-time curves are presented in Fig. 3, and the calculated pharmacokinetic parameters are shown in Table 3. The $\mathrm{T}_{\max }$ of propofol released by HX0969W ( propofol $_{\mathrm{H}}, 4.0 \pm 0.47 \mathrm{~min}$ ) or fospropofol disodium ( propofol $_{\mathrm{F}}, 4.5 \pm 1.18 \mathrm{~min}$ ) in plasma had no significant difference, which was longer than that for propofol $(0.5 \mathrm{~min})$. However, the $\mathrm{C}_{\max }$ of $\operatorname{propofol}_{\mathrm{H}}(24.26 \pm 5.14 \mu \mathrm{g} / \mathrm{ml})$ was similar to the parent drug propofol $(23.4 \pm 4.69 \mu \mathrm{g} / \mathrm{ml})$, which was higher than that for $\operatorname{propofol}_{\mathrm{F}}(21.91 \pm 4.98 \mu \mathrm{g} / \mathrm{ml})$. The AUC, MRT, and CL of $\operatorname{propofol}_{\mathrm{F}}(740.5 \pm 186.33$ 
$212 \mathrm{~min} * \mu \mathrm{g} / \mathrm{ml}, 52.6 \pm 2.90 \mathrm{~min}$, and $54.67 \pm 13.83 \mathrm{ml} / \mathrm{min} / \mathrm{kg}$, respectively) were similar to those of

213 propofol $_{\mathrm{H}}(704.53 \pm 226.25 \mathrm{~min} * \mu \mathrm{g} / \mathrm{ml}, 50.92 \pm 4.40 \mathrm{~min}$, and $68.12 \pm 22.84 \mathrm{ml} / \mathrm{min} / \mathrm{kg}$,

214 respectively), but were higher than those of propofol $(219.23 \pm 42.21 \mathrm{~min} * \mu \mathrm{g} / \mathrm{ml}, 29.45 \pm 4.63$

$215 \mathrm{~min}$, and $43.28 \pm 8.46 \mathrm{ml} / \mathrm{min} / \mathrm{kg}$, respectively). In this pharmacokinetic study, the longer onset

216 time and duration of HX0969W and fospropofol disodium, than those of the parent drug

217 propofol, were due to their pharmacokinetic features, which included a larger AUC and a longer

218 MRT. Furthermore, the onset time and duration of HX0969W and fospropofol disodium, which

219 showed no significant difference, could be related to the undifferentiated pharmacokinetic

220 features of propofol $\mathrm{H}_{\mathrm{H}}$ and propofol $\mathrm{F}_{\mathrm{F}}$, including $\mathrm{T}_{\max }, \mathrm{C}_{\max }, \mathrm{AUC}, \mathrm{MRT}$ and CL.

\section{4. Discussion}

In this study, we have demonstrated that HX0969W requires a longer time to induce the sedative-hypnotic effect, but with longer duration than propofol in rats. HX0969W had a large

$\mathrm{C}_{\max }$, a short MRT, and a rapid $\mathrm{CL}$ than fospropofol disodium in rats. The $\mathrm{T}_{\max }$ of propofol

released by HX0969W or fospropofol disodium showed no significant difference, and the $\mathrm{C}_{\max }$

of propofol released by HX0969W was similar to that of propofol, which was higher than that of

the propofol released by fospropofol disodium.

The main objective of this study was to develop a new water-soluble pro-drug of propofol

with non-toxic metabolites. In the pharmacodynamic study, the potency of HX0969W was found

to be similar to that of fospropofol disodium, but lower than that of the parent drug propofol.

231 Similar to fospropofol disodium, the onset time and duration of HX0969W after single 
water-soluble pro-drug of propofol that can be used for induction and maintenance of general

anesthesia. In contrast to fospropofol disodium, which is metabolized into a toxic metabolite,

HX0969W was metabolized to propofol, GHB, and phosphate in vivo; GHB is further converted

into carbon dioxide and water (Lang et al. 2014; Rousseau et al. 2012). Previous studies have

demonstrated that GHB is mainly used as a sedative drug for children suffering from burns or for

the treatment of alcohol and opioid addiction (Brambilla et al. 2012; Gallimberti et al. 1993;

Rousseau et al. 2012). However, it was reported that GHB produced an obvious hypnotic effect

after oral administration at least $800 \mathrm{mg} / \mathrm{kg}$ (Lettieri \& Fung 1979). In our published study,

HX0969W produced a sedative-hypnotic effect after oral administration with $193.08 \mathrm{mg} / \mathrm{kg}$ in

rats (Wang et al. 2015), which was far less than $800 \mathrm{mg} / \mathrm{kg}$. Meanwhile, there is no data to show

if there is any synergy between the effects of propofol and GHB. Therefore, the LoRR caused by

HX0969W administration was mainly caused by the propofol released by HX0969W. As with lesser potential risks of systemic toxicity.

hypotension $(n=4,3.3 \%)$, bradycardia $(n=1,0.8 \%)$. No patient experienced apnea during the 
$25436,14.3 \%)$ and hypotension $(n=8,3.2 \%)$. In another clinical trial (Cohen 2008), four patients in

255 fospropofol group experienced sedation-related adverse events: mild hypotension $(\mathrm{n}=2,2 \%)$

256 and hypoxemia $(n=2,2 \%)$. Only one patient of hypoxemia required the airway assistance

257 (verbal stimulation). Thus, fospropofol showed lower incidences of hypotension, respiratory

258 depression, apnea, and loss of airway patency because of its slower onset of action (Mahajan et

259 al. 2012). On the basis of the findings in this study, there is no significant difference between

260 HX0969W and fospropofol disodium in pharmacodynamic and pharmacokinetic studies.

261 Therefore, it would be reasonable to consider the possibility that HX0969W could reduce the

262 cardiopulmonary side effects by a slow-released propofol as fospropofol disodium. In the

263 subsequent experiments, we will focus on evaluating the safety of HX0969W and propofol in the

264 cardiovascular and respiratory systems.

265 Propofol has been the most widely used intravenous anesthetic in clinical practices due to

266 its rapid onset and recovery from sedation (Hemphill et al. 2019). However, the pharmacokinetic

267 features of $\mathrm{HX} 0969 \mathrm{~W}$, including a longer MRT and $\mathrm{t}_{1 / 2}$, and a larger AUC, have superseded the

268 advantages of propofol. Therefore, HX0969W is more appropriate for patients undergoing

269 longstanding surgeries or a long-term sedation in intensive care unit, and its use can avoid the

270 side effects of propofol by its lipid emulsion preparation. Meanwhile, HX0969W generated a

271 hypnotic effect with rapid onset and shorter duration than fospropofol disodium and propofol

272 after oral administration in rats (Wang et al. 2015). Therefore, there might be a new clinical

273 indication for HX0969W for use in patients undergoing pre-operative sedation, transitory

274 diagnostic or therapeutic procedures by oral administration. Above all, our results show that 
275 HX0969W can avoid the disadvantages associated with the lipid emulsion formulation of

276 propofol. Thus, HX0969W is more suitable for long-term sedation and pre-operative preparation

277 for children who do not cooperate with venipuncture.

\section{5. Conclusions}

279 With the equivalent dose, HX0969W and fospropofol disodium had a longer time to cause LoRR

with longer duration than propofol in rats. In pharmacokinetic study, the $\mathrm{C}_{\max }$ of fospropofol

disodium was higher than HX0969W. HX0969W had a shorter MRT and a rapid CL than

fospropofol disodium. Due to the pro-drug design, HX0969W and fospropofol disodium are

metabolized to propofol, which produces the sedative-hypnotic effect. The $\mathrm{T}_{\max }$ of propofol

released by HX0969W and fospropofol disodium had no significant difference, which was

longer than that for propofol. The $\mathrm{C}_{\max }$ of propofol released by HX0969W was similar to the

parent drug propofol, which was higher than that for that of propofol released by fospropofol

287 disodium.

288 Acknowledgments:

The authors would like to thank Linghui Yang and Deying Gong (Laboratory of Anaesthesia and

Critical Care Medicine, Translational Neuroscience Centre, West China Hospital, Sichuan

University, Chengdu, China) for the assistance in reviewing the manuscript. Meanwhile, we are also grateful to LinQiao Tang and Yan Wang from the Core facility of West China Hospital for their technical supports.

\section{References}


mid/long term treatment of alcohol dependence: a systematic review]. Riv Psichiatr 47:269-280. 10.1708/1139.12554

Cohen LB. 2008. Clinical trial: a dose-response study of fospropofol disodium for moderate sedation during colonoscopy. Aliment Pharmacol Ther 27:597-608. 10.1111/j.1365-2036.2008.03598.x

Cohen LB, Cattau E, Goetsch A, Shah A, Weber JR, Rex DK, and Kline JM. 2010. A randomized, double-blind, phase 3 study of fospropofol disodium for sedation during colonoscopy. J Clin Gastroenterol 44:345-353. 10.1097/MCG.0b013e3181c2987e

Diaz JH, Prabhakar A, Urman RD, and Kaye AD. 2014. Propofol infusion syndrome: a retrospective analysis at a level 1 trauma center. Crit Care Res Pract 2014:346968. 10.1155/2014/346968

Dixon WJ. 1991. Staircase bioassay: the up-and-down method. Neurosci Biobehav Rev 15:47-50.

Feng AY, Kaye AD, Kaye RJ, Belani K, and Urman RD. 2017. Novel propofol derivatives and implications for anesthesia practice. J Anaesthesiol Clin Pharmacol 33:9-15. 10.4103/0970-9185.202205

Gallimberti L, Cibin M, Pagnin P, Sabbion R, Pani PP, Pirastu R, Ferrara SD, and Gessa GL. 1993. Gammahydroxybutyric acid for treatment of opiate withdrawal syndrome. Neuropsychopharmacology 9:77-81. 10.1038/npp.1993.45

Gan TJ, Berry BD, Ekman EF, Muckerman RC, 2nd, Shore N, and Hardi R. 2010. Safety evaluation of fospropofol for sedation during minor surgical procedures. J Clin Anesth 22:260-267. 10.1016/j.jclinane.2009.08.007

Garnock-Jones KP, and Scott L. 2010. Fospropofol. Drugs 70:469-477.

Glen JB, and Hunter SC. 1984. Pharmacology of an emulsion formulation of ICl 35 868. Br J Anaesth 56:617-626.

Hemphill S, McMenamin L, Bellamy MC, and Hopkins PM. 2019. Propofol infusion syndrome: a structured literature review and analysis of published case reports. $\mathrm{Br} J$ Anaesth 122:448-459. 10.1016/j.bja.2018.12.025

Kilpatrick GJ, McIntyre MS, Cox RF, Stafford JA, Pacofsky GJ, Lovell GG, Wiard RP, Feldman PL, Collins H, Waszczak BL, and Tilbrook GS. 2007. CNS 7056: a novel ultra-short-acting Benzodiazepine. Anesthesiology 107:60-66. 10.1097/01.anes.0000267503.85085.c0

Kumpulainen H, Jarvinen T, Mannila A, Leppanen J, Nevalainen T, Mantyla A, Vepsalainen J, and Rautio J. 2008. Synthesis, in vitro and in vivo characterization of novel ethyl dioxy phosphate prodrug of propofol. Eur J Pharm Sci 34:110-117. 10.1016/j.ejps.2008.02.121

Lang BC, Yang J, Wang Y, Luo Y, Kang Y, Liu J, and Zhang WS. 2014. An improved design of water-soluble propofol prodrugs characterized by rapid onset of action. Anesth Analg 118:745-754. 10.1213/ANE.0000000000000124

Lee JH, Jung SY, Kim MH, and Cho K. 2014. The effect of dexmedetomidine on propofol injection pain. Korean J Anesthesiol 67:S30-31. 10.4097/kjae.2014.67.S.S30

Lettieri JT, and Fung HL. 1979. Dose-dependent pharmacokinetics and hypnotic effects of sodium gammahydroxybutyrate in the rat. J Pharmacol Exp Ther 208:7-11.

Mahajan R, Mahajan B, and Kaushal S. 2012. Fospropofol. Journal of Pharmacology and Pharmacotherapeutics 3. 10.4103/0976-500x.99457

Mirrakhimov AE, Voore P, Halytskyy O, Khan M, and Ali AM. 2015. Propofol infusion syndrome in adults: a clinical update. Crit Care Res Pract 2015:260385. 10.1155/2015/260385

Moore GD, Walker AM, and MacLaren R. 2009. Fospropofol: a new sedative-hypnotic agent for monitored anesthesia care. Ann Pharmacother 43:1802-1808. 10.1345/aph.1M290 
337

338

339

340

341

342

343

344

345

346

347

348

349

350

351

352

353

354

355

356

357

358

359

360

Pestana D, Garcia-de-Lorenzo A, and Madero R. 1996. Metabolic pattern and lipid oxidation during abdominal surgery: midazolam versus propofol. Anesth Analg 83:837-843. 10.1097/00000539-199610000-00032

Prankerd RJ, and Stella VJ. 1990. The use of oil-in-water emulsions as a vehicle for parenteral drug administration. $J$ Parenter Sci Technol 44:139-149.

Rousseau AF, Ledoux D, Sabourdin N, Richard P, Damas P, and Constant I. 2012. Clinical sedation and bispectral index in burn children receiving gamma-hydroxybutyrate. Paediatr Anaesth 22:799-804. 10.1111/j.14609592.2011.03756.x

Silvestri GA, Vincent BD, Wahidi MM, Robinette E, Hansbrough JR, and Downie GH. 2009. A phase 3, randomized, double-blind study to assess the efficacy and safety of fospropofol disodium injection for moderate sedation in patients undergoing flexible bronchoscopy. Chest 135:41-47. 10.1378/chest.08-0623

Singh DK, Jindal P, and Singh G. 2011. Comparative study of attenuation of the pain caused by propofol intravenous injection, by granisetron, magnesium sulfate and nitroglycerine. Saudi J Anaesth 5:50-54. 10.4103/1658-354X.76511

Telletxea S, Lauzirika Z, Etxebarria A, and Ortega LF. 2012. [Fospropofol: A new prodrug of propofol]. Rev Esp Anestesiol Reanim 59:497-502. 10.1016/j.redar.2012.05.033

Wachowski I, Jolly DT, Hrazdil J, Galbraith JC, Greacen M, and Clanachan AS. 1999. The growth of microorganisms in propofol and mixtures of propofol and lidocaine. Anesth Analg 88:209-212.

Wang HY, Yang J, Yin W, Yang LH, and Zhang WS. 2015. [The sedative-hypnotic effects and safety of oral administrated propofol prodrugs hx0969w and fospropofol disodiun in comparison with propofol emulsion in rats]. Sichuan Da Xue Xue Bao Yi Xue Ban 46:214-217.

Wolf A, Weir P, Segar P, Stone J, and Shield J. 2001. Impaired fatty acid oxidation in propofol infusion syndrome. Lancet 357:606-607. 10.1016/S0140-6736(00)04064-2

Zhou ZB, Yang XY, Zhou X, Niu L, Xiao LC, Huang WQ, and Feng X. 2015. Propofol-induced rhabdomyolysis: a case report. Int J Clin Pharmacol Ther 53:890-894. 10.5414/CP202341 


\section{Figure 1}

Figure 1 The onset time (A) and duration (B) of hypnotic behavior observation after intravenous administration of 2 -fold $\mathrm{ED}_{50}$ drugs ( $\mathrm{n}=10$ in each group).

Data are expressed as mean \pm SD. $* p<0.05 ; \# p<0.01$.

A

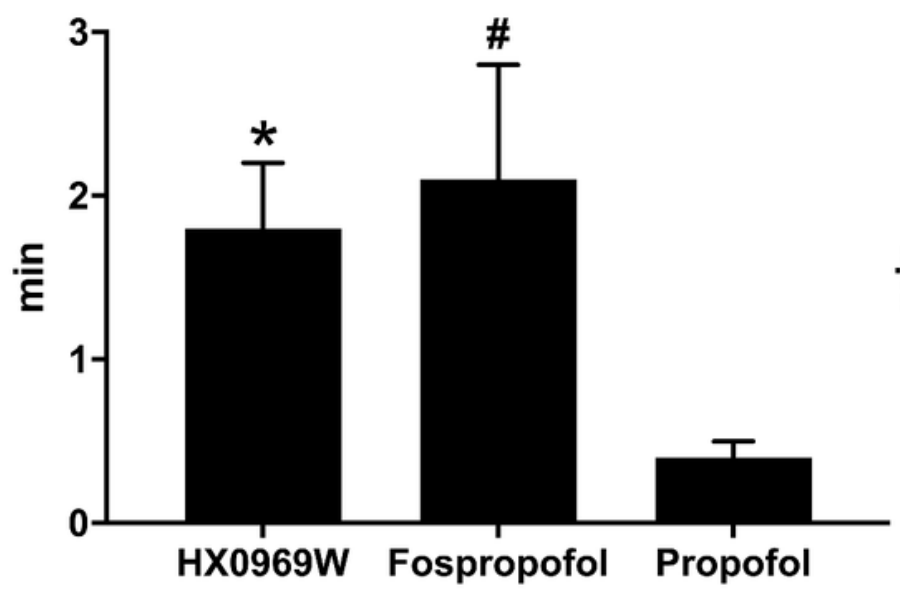

B Time to recovery form LoRR

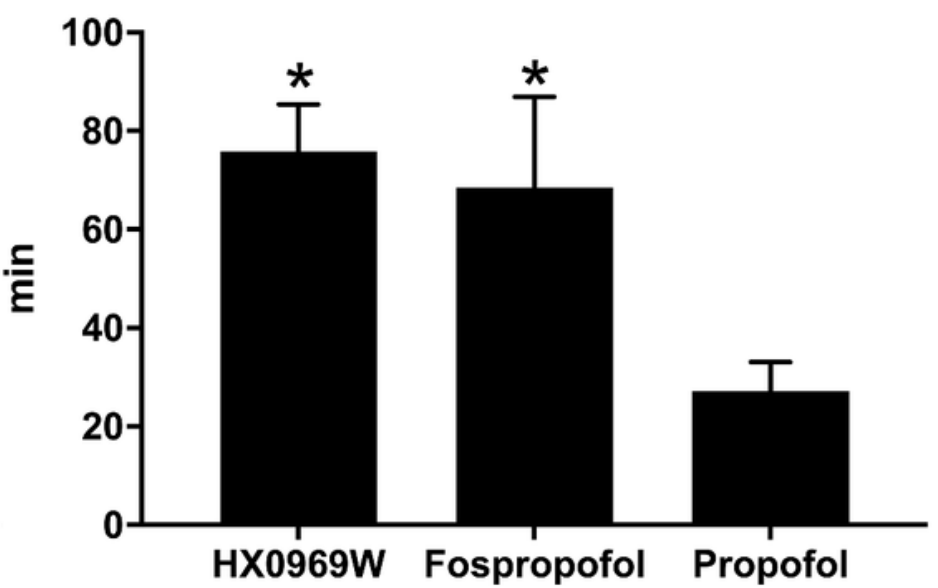


Figure 2

Figure 2 Mean concentration-time profiles of HX0969W (A) and fospropofol disodium (B) in plasma after intravenous administration with of 2 -fold $\mathrm{ED}_{50}$ in rats $(n=10$ in each group).

Data are expressed as mean \pm SD. 

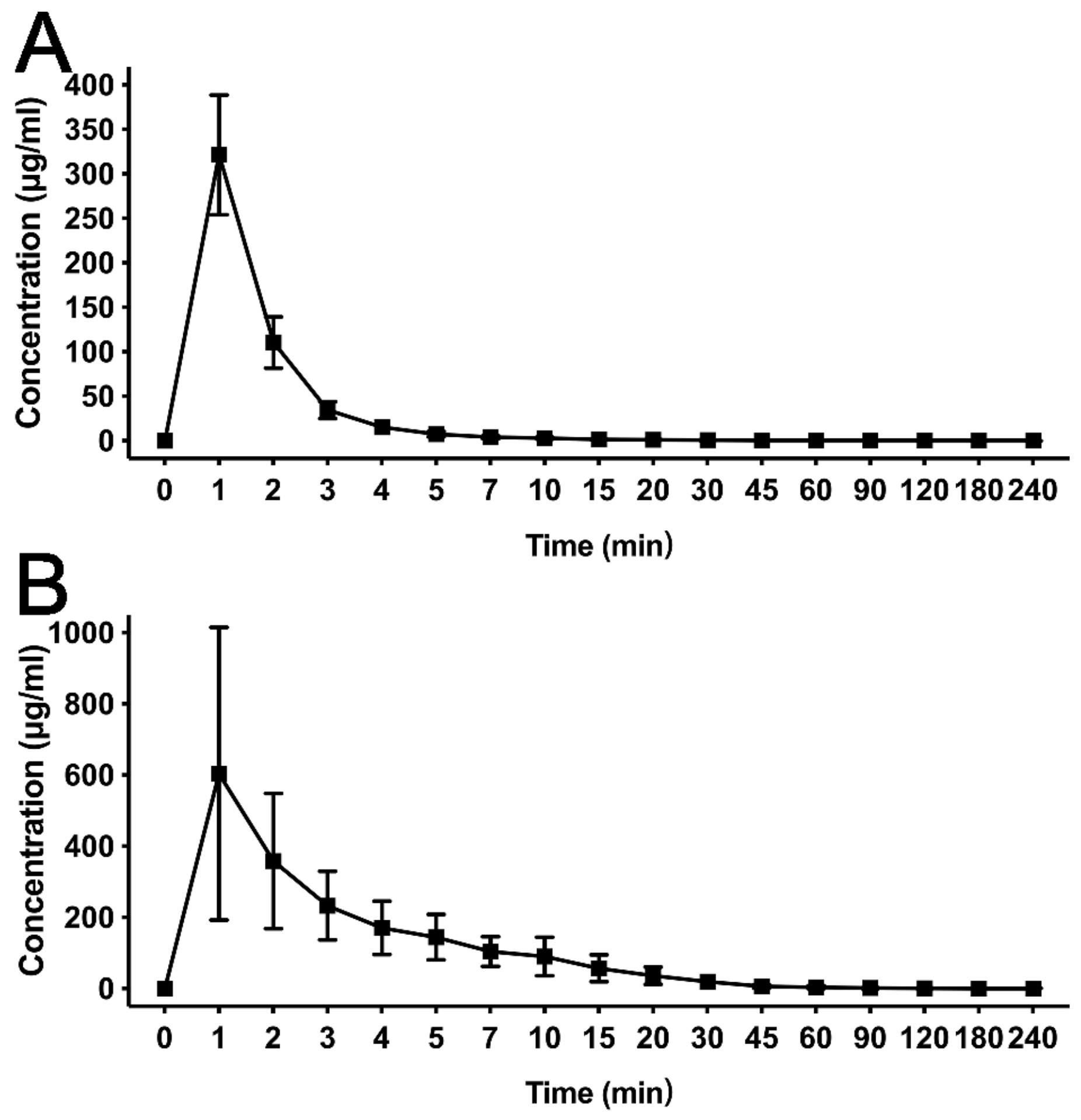
Figure 3

Figure 3 Mean concentration-time profiles of propofol in plasma after intravenous

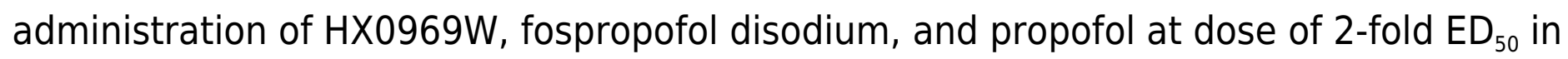
rats ( $n=10$ in each group).

Data are expressed as mean \pm SD. Propofol ${ }_{H}$, propofol released by HX0969W; Propofol ${ }_{F}$, propofol released by fospropofol disodium.

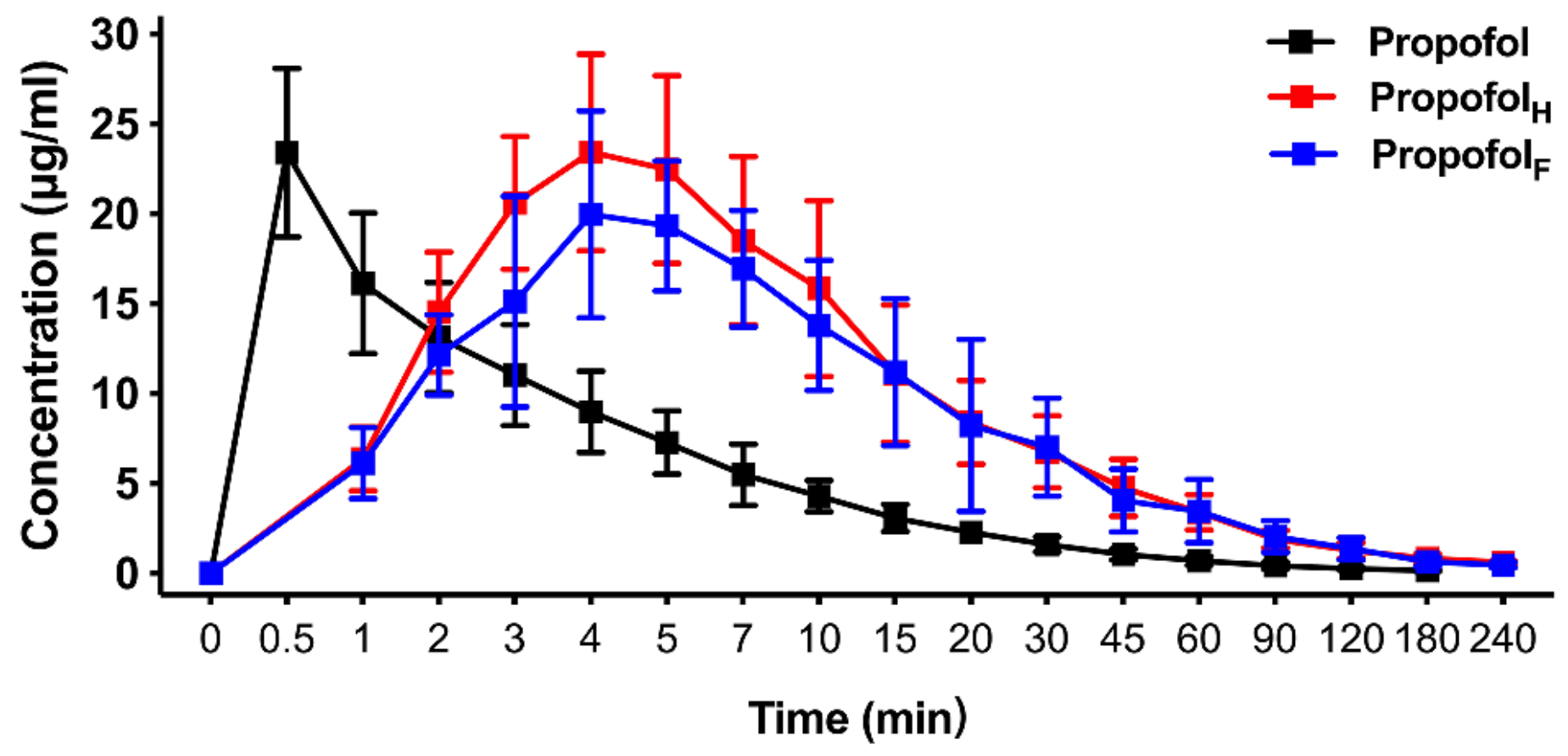




\section{Table $\mathbf{1}$ (on next page)}

Calculation process of $\mathrm{ED}_{50}$ for $\mathrm{HX0969W}$, fospropofol disodium and propofol

$1, X=\lg ($ dose $) ; t=$ sum total of rat; $C=X \times t ; M=X^{2} \times t$.

$2,+$ : rat with loss of the righting reflex; -: rat without loss of the righting reflex.

$3,95 \% \mathrm{Cl}=\lg ^{-1}\left(\operatorname{lgED}_{50} \pm 1.96 \mathrm{slgED}_{50}\right) ; \operatorname{SlgED}_{50}=\left\{\left[\Sigma \mathrm{M}-(\Sigma \mathrm{C})^{2} / \Sigma \mathrm{t}\right] /(\Sigma \mathrm{t} \times(\Sigma \mathrm{t}-1)\} 1 / 2\right.$. 


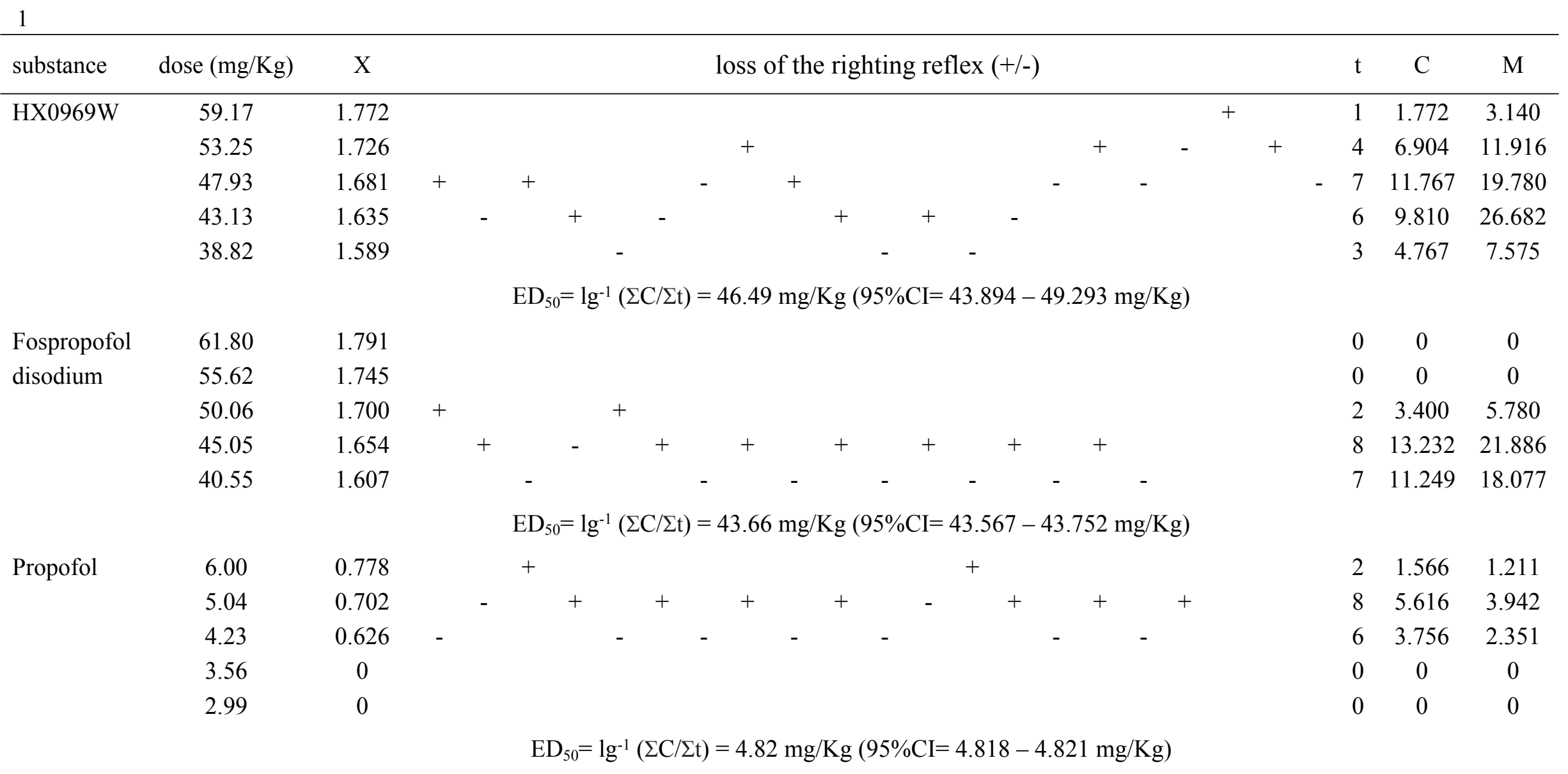


Table 2 (on next page)

Pharmacokinetic parameters of HX0969W and fospropofol (mean \pm SD).

AUC, area under the curve; MRT, mean residual time; $C L$, clearance. 
1

\begin{tabular}{lcc}
\hline Parameters & HX0969W & fospropofol \\
\hline $\mathrm{C}_{\max }(\mu \mathrm{g} / \mathrm{ml})$ & $321.3 \pm 67.22$ & $603.49 \pm 411.29$ \\
$\mathrm{t}_{1 / 2}(\mathrm{~min})$ & $71.79 \pm 59.52$ & $49.29 \pm 40.31$ \\
$\mathrm{AUC}_{0 \sim \mathrm{t}}\left(\mathrm{min}^{*} \mu \mathrm{g} / \mathrm{ml}\right)$ & $1053.78 \pm 214.37$ & $3804.92 \pm 2091.75$ \\
$\mathrm{MRT}(\mathrm{min})$ & $3.67 \pm 1.71$ & $13.15 \pm 5.45$ \\
$\mathrm{CL}(\mathrm{ml} / \mathrm{min} / \mathrm{kg})$ & $89.97 \pm 15.94$ & $31.12 \pm 19.09$ \\
\hline
\end{tabular}

2 


\section{Table 3(on next page)}

Pharmacokinetic parameters of propofol (mean \pm SD).

${ }^{1}$ Propofol $_{H}$, propofol released by HX0969W; Propofol ${ }_{\mathrm{F}}$, propofol released by fospropofol.

${ }^{2} \mathrm{AUC}$, area under the concentration; MRT, mean residual time; $\mathrm{CL}$, clearance. 
1

\begin{tabular}{lccc}
\hline Parameters & Propofol & Propofol $_{\mathrm{H}}$ & Propofol $_{\mathrm{F}}$ \\
\hline $\mathrm{C}_{\max }(\mu \mathrm{g} / \mathrm{ml})$ & $23.4 \pm 4.69$ & $24.26 \pm 5.14$ & $21.91 \pm 4.98$ \\
$\mathrm{~T}_{\max }(\mathrm{min})$ & 0.5 & $4.0 \pm 0.47$ & $4.5 \pm 1.18$ \\
$\mathrm{t}_{1 / 2}(\mathrm{~min})$ & $54.24 \pm 16.18$ & $88.62 \pm 77.35$ & $78.97 \pm 29.05$ \\
$\mathrm{AUC}_{0 \sim \mathrm{t}}\left(\mathrm{min}^{*} \mu \mathrm{g} / \mathrm{ml}\right)$ & $219.23 \pm 42.21$ & $740.5 \pm 186.33$ & $704.53 \pm 226.25$ \\
$\mathrm{MRT}(\mathrm{min})$ & $29.45 \pm 4.63$ & $52.6 \pm 2.90$ & $50.92 \pm 4.40$ \\
$\mathrm{CL}(\mathrm{ml} / \mathrm{min} / \mathrm{kg})$ & $43.28 \pm 8.46$ & $54.67 \pm 13.83$ & $68.12 \pm 22.84$ \\
\hline
\end{tabular}

2 